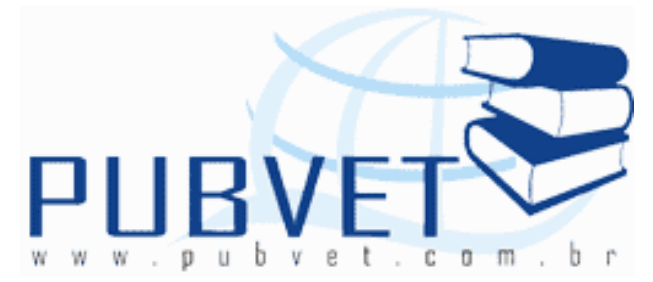

PUBVET, Publicações em Medicina Veterinária e Zootecnia.

\title{
Intoxicação experimental de cães com folhas verdes de Nerium oleander e uso da frutose 1,6 difosfato e da glicose como tratamentos
}

\author{
Carolina Bellodi ${ }^{1}$, José Javier Mesa Socha ${ }^{2}$, Mario Roberto Hatayde ${ }^{3}$ \\ ${ }^{1}$ Doutoranda Programa de Medicina Veterinária, FCAV-UNESP, SP, Brasil. \\ E-mail:carolbellodi@hotmail.com \\ 2 Médico Veterinário autônomo, Bogotá,Colombia. \\ 3 Professor Departamento de Clínica e Cirurgia Veterinária, FCAV-UNESP, \\ Jaboticabal, SP, Brasil.
}

\section{Resumo}

O N. oleander é uma planta com ampla distribuição mundial, principalmente em regiões tropicais e subtropicais. Esses arbustos são frequentemente usados como plantas ornamentais e possuem mais de 30 glicosídeos cardíacos causadores do quadro clinico de intoxicação em caninos. Sabendo-se disso, nosso estudo objetivou-se a avaliar as alterações clinicas, eletrocardiográficas, bioquímicas e hematológicas do rim e também promover um possível protocolo terapêutico avaliando o uso da frutose 1,6 difosfato e da glicose nos animais intoxicados experimentalmente. Os cães apresentaram sinais clínicos de intoxicação como vômito, sialorréia, náuseas, apatia, conjuntiva ocular congesta, desidratação, dor abdominal, tremores, diarreia, inapetência e tenesmo. Observou-se nos exames bioquímicos elevação principalmente da GGTU, CK e CKMB. Pela análise do eletrocardiograma encontraram-se arritmias 
BELLODI, C., SOCHA, J.J.M. e HATAYDE, M.R. Intoxicação experimental de cães com folhas verdes de Nerium oleander e uso da frutose 1,6 difosfato e da glicose como tratamentos. PUBVET, Londrina, V. 8, N. 5, Ed. 254, Art. 1679, Março, 2014.

como: bradicardia sinusal, bloqueios atrioventriculares de segundo grau, taquicardia ventricular paroxístico e complexo ventricular prematuro. Nos tratamentos, observou-se uma melhora significativa no grupo tratado com frutose 1,6 difosfato. Concluiu-se com o presente estudo que os animais intoxicados com $0,25 \mathrm{~g} / \mathrm{kg}$ de folhas verdes de Nerium oleander em única dose, causa sinais clínicos, alterações laboratoriais e mudanças no traçado do eletrocardiograma.

Palavras-chave: arritmia, glicosídeos, biópsia, eletrocardiografia, intoxicação.

\title{
Experimental poisoning of dogs with green leaves of Nerium oleander and use of fructose 1,6 diphosphate and glucose as treatments
}

\begin{abstract}
The N. oleander is a plant with worldwide distribution, especially in tropical and subtropical regions. These shrubs are often used as ornamental plants and have more than 30 cardiac glycosides cause the clinical picture of poisoning in canines. Knowing this, our study aimed to assess the changes clinical, electrocardiographic, biochemical and hematological kidney and also promote a possible therapeutic protocol evaluating the use of fructose 1,6 diphosphate and glucose in animals experimentally poisoned. Dogs showed clinical signs of poisoning such as vomiting, salivation, nausea, listlessness, ocular conjunctiva congested, dehydration, abdominal pain, tremors, diarrhea, loss of appetite and tenesmus. Was observed an increase of the biochemical GGTU, CK and CK-MB. By analyzing the electrocardiogram met arrhythmias as sinus bradycardia, second degree atrioventricular blocks, ventricular tachycardia and paroxysmal ventricular premature complex. Treatments, there was a significant improvement in the group treated with fructose 1,6 diphosphate. Concluded with this study that the animals intoxicated with $0.25 \mathrm{~g} / \mathrm{kg}$ of green leaves of Nerium oleander in single dose, because clinical signs, laboratory findings and changes in the electrocardiogram tracing.
\end{abstract}

Keywords: arrhythmia, glycosides, biopsy, electrocardiography, intoxication. 
BELLODI, C., SOCHA, J.J.M. e HATAYDE, M.R. Intoxicação experimental de cães com folhas verdes de Nerium oleander e uso da frutose 1,6 difosfato e da glicose como tratamentos.

PUBVET, Londrina, V. 8, N. 5, Ed. 254, Art. 1679, Março, 2014.

\section{Introdução}

As intoxicações tornam-se mais frequentes, apontando um notório acréscimo da casuística na rotina clínica de pequenos animais, principalmente pelo uso de produtos domissanitários e uso de plantas ornamentais tóxicas. Tanto os cães como os gatos podem potencialmente consumir as plantas ou parte dessas, especialmente os filhotes devido a sua curiosidade. Em adultos a ingestão é causada por desordens comportamentais ou em animais com restrição alimentar. O Nerium oleander é amplamente usada como planta ornamental e provoca desordens cardiovasculares devido aos glicosídeos cardíacos. Glicosídeos cardíacos esses que estão presentes em 45 espécies de plantas diferentes que são tóxicos para animais e humanos (EVERIST, 1982). Plantas tóxicas geralmente desencadeiam quadros clínicos inespecíficos que podem ser confundidos com diversos tipos de intoxicação ou com outras doenças, que poderia dificultar seu diagnóstico, somado ao desconhecimento que existe, nos mecanismos de ação, fisiopatologia, princípios ativos e terapêuticos, existentes nesses tipos de intoxicação. Por esse motivo, atualmente vem-se realizando diversas pesquisas a respeito, na tentativa de dispor ferramentas de estudo aos médicos veterinários e novas possibilidades de tratamento.

Nerium Oleander é um arbusto amplamente utilizado como planta ornamental e sua importância se da principalmente por sua ampla distribuição em regiões tropicais e subtropicais do globo terrestre. Pertencente à família Apocynaceae, se caracterizando como arbustos perenes, folhas lanceoladas dispostas em espiral de coloração variando do amarelo ao verde escuro com florescência em todos os meses do verão. Conhecido popularmente como espirradeira, oleandro rosa, baladre, laurel de flor (LANGFORD; BOOR, 1996).

Ainda que a planta seja reportada como pouco palatável, ela pode ser ingerida por animais famintos ou entediados causando a intoxicação (LYNN; SAFDAR, 2006), pelos seus glicosídeos cardíacos os quais são quimicamente similares com a digoxina. Esses glicosídeos cardíacos podem ser encontrados em todas as partes da planta fresca ou seca (LANGFORD; BOOR, 1996; SOTO- 
BELLODI, C., SOCHA, J.J.M. e HATAYDE, M.R. Intoxicação experimental de cães com folhas verdes de Nerium oleander e uso da frutose 1,6 difosfato e da glicose como tratamentos. PUBVET, Londrina, V. 8, N. 5, Ed. 254, Art. 1679, Março, 2014.

BLANCO et al., 2006), podendo verificar-se até 30 tipos diferentes como as oleandrinas, digitoxigeninas e folinerinas (IBRAHIM et al., 2008).

O contato da planta com a pele e mucosas causam sérios problemas como eritema bucal, dermatite por contato, náuseas, vomito, sialorréia, dores abdominais, cefaleia, alterações do estado mental, distúrbios visuais, midríase, neurites e principalmente sintomas cardiovasculares (HUGHES et al., 2002; ASLANI et al., 2007). Os sintomas cardiovasculares que são dados pela presença dos glicosídeos cardíacos na planta, estruturalmente são similares a estrutura da digoxina e sua ação esta ligada diretamente a inibição da bomba de sódio e potássio ATPase ( $\mathrm{Na} / \mathrm{K}$-ATPase), resultando na depleção intracelular de potássio e aumento do sódio intracelular. Essas alterações acarretam no acumulo citoplasmático de cálcio, responsável pelo efeito inotrópico positivo. Esse distúrbio eletrolítico afeta a condutividade elétrica do coração (LANGFORD; BOOR, 1996). Nessas condições, a resposta simpática também aumenta, sensibilizando o miocárdio e exacerbando todos os efeitos tóxicos dos glicosídeos (JOUBERT, 1989; ASLANI et al., 2004). Devido a redução da condutividade elétrica no miocárdio, são observados bloqueio de condução, tais como arritmias ventriculares e eventualmente a perda completa da contratilidade (IBRAHIM et al., 2008).

Quanto ao diagnóstico das intoxicações por plantas que contém glicosídeos cardíacos, este envolve a identificação da planta e a evidência do consumo desta, informações que são obtidas através da história clínica, sinais clínicos, achados do eletrocardiograma, alterações clínico-patológicas e achados de necropsia, embora esses parâmetros muitas vezes não sejam suficientes para concluir o diagnóstico (HUGHES et al., 2002).

$\mathrm{Na}$ medicina humana, o radioimunoensaio (RIA) é utilizado para o monitoramento das concentrações séricas das digoxinas e também para confirmar o envenenamento fatal em seres humanos por Thevetia peruviana (BREWSTER, 1986) e Nerium oleander (MARTINEZ et al., 2007; MARIN et al., 2007), já que os glicosídeos especialmente a oleandrina, cuja estrutura é similar a digoxina, apresentam reatividade cruzada com o exame RIA 
BELLODI, C., SOCHA, J.J.M. e HATAYDE, M.R. Intoxicação experimental de cães com folhas verdes de Nerium oleander e uso da frutose 1,6 difosfato e da glicose como tratamentos.

PUBVET, Londrina, V. 8, N. 5, Ed. 254, Art. 1679, Março, 2014.

(CHEUNG et al., 1989; JORTANI et al., 1996). A cromatografia líquida por espectrofotometria de massa pode ser utilizada em alguns casos, na avaliação do conteúdo gastrintestinal, fezes e sangue de animais e humanos (GALEY, 1996; IBRAHIM et al., 2008), mas por sue alto custo não é utilizada frequentemente na medicina veterinária (HUGHES et al., 2002).

O tratamento da intoxicação por $\mathrm{N}$. oleander consiste em dar suporte com fluidoterapia via oral ou intravenosa para diminuir os efeitos cardiovasculares (HUGHES et al., 2002). Os fluidos que contém cálcio devem ser evitados, pois podem aumentar o efeito dos glicosídeos cardíacos no miocardio (KNIGHT, 1988). Também doses repetidas de carvão ativado são empregadas para prevenir a reciclagem de toxinas entero-hepáticas (LYNN et al., 2006). Os medicamentos antiarrítmicos como a atropina, atenolol, fentoína, procainamida e lidocaína podem ser usados para controlar os efeitos dos glicosídeos cardíacos (JOUBERT, 1989; PLUMB, 1999). Contudo, deve-se constatar o tipo da arritmia, realizando o exame eletrocardiográfico durante vários dias (LANGFORD; BOOR, 1996).

Alem do tratamento suporte, foi demonstrado experimentalmente que a frutose-1,6 difosfato, previne danos no coração e o efeito de desbalanceamento dos íons em cães, ainda que o mecanismo de ação seja desconhecido. Cães intoxicados com extratos secos de N. oleander, foram tratados com dosagens de $50 \mathrm{mg} / \mathrm{kg}$ de frutose-1,6 difosfato e em seguida, utilizada uma solução a $10 \%$ do mesmo princípio em infusão constante, demonstrando que o coração dos animais tratados com frutose-1,6 disfosfato voltaram ao ritmo normal, os níveis de potássio nos cães não sofreram alterações e a pressão arterial foi mantida no nível da normalidade (MARKOV et al., 1999). Também outra opção na terapêutica é a utilização de fragmentos de anticorpos de digoxina bem sucedida no tratamento experimental de intoxicação com N. oleander em cães (CLARCK et al., 1991; CAMPHAUSEN et al., 2005). O objetivo geral desse trabalho foi avaliar as alterações clínicas, eletrocardiográficas, bioquímicas e hematológicas dos cães intoxicados com 
BELLODI, C., SOCHA, J.J.M. e HATAYDE, M.R. Intoxicação experimental de cães com folhas verdes de Nerium oleander e uso da frutose 1,6 difosfato e da glicose como tratamentos. PUBVET, Londrina, V. 8, N. 5, Ed. 254, Art. 1679, Março, 2014.

$0,25 \mathrm{~g} / \mathrm{Kg}$ de folhas secas de Nerium oleander que foram trituradas e adicionadas a ração em uma única dose.

\section{Material e métodos}

Foram utilizados dez cães adultos, hígidos, machos e fêmeas, sem raça definida, pesando entre $10-25 \mathrm{Kg}$ provenientes do centro de pesquisa, parasitologia e sanidade animal- CPPAR- Unesp - Jaboticabal. Esses foram alojados individualmente em canis equipados com comedouros e bebedouros individuais, localizados no hospital veterinário "Governador Laudo Natel"Unesp - Jaboticabal.

Todos os animais receberam a mesma dieta (ração comercial) durante o período experimental. Os cães foram divididos em dois grupos compostos por cinco animais, (G1 e G2) delineados inteiramente ao acaso. Os dois grupos receberam uma única dose de $0,25 \mathrm{~g} / \mathrm{Kg}$ de folhas verdes de $\mathrm{N}$. oleander, juntamente a ração e depois avaliados da seguinte maneira seguindo a figura 1 descrita a seguir:

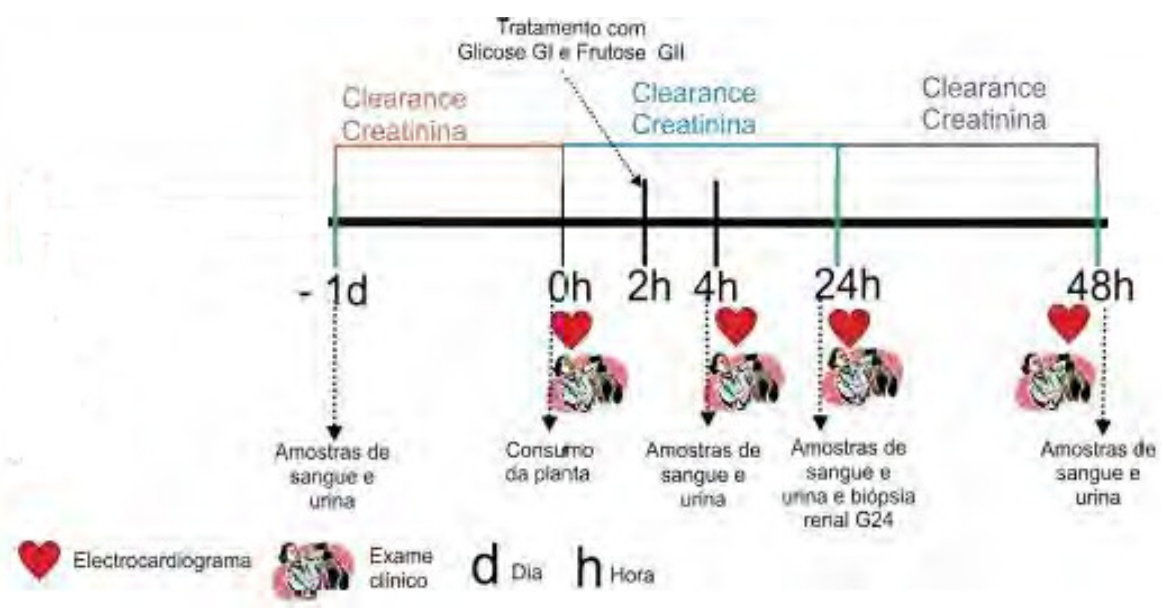

Figura 1. Ilustração dos intervalos experimentais, indicando tempo e procedimentos realizados. 
BELLODI, C., SOCHA, J.J.M. e HATAYDE, M.R. Intoxicação experimental de cães com folhas verdes de Nerium oleander e uso da frutose 1,6 difosfato e da glicose como tratamentos. PUBVET, Londrina, V. 8, N. 5, Ed. 254, Art. 1679, Março, 2014.

Os animais foram observados e avaliados clinicamente 24 horas préintoxicação e até 48 horas pós-intoxicação. Foi estabelecido tempo zero (controle) tomados como dados basais de cada animal, antes do fornecimento das folhas verdes de $\mathrm{N}$. oleander para a realização do eletrocardiograma, exames hematológicos e bioquímicos.

Para os tratamentos, foram utilizados dois protocolos diferentes. Em um grupo foi utilizada glicose $50 \mathrm{mg} / \mathrm{Kg}$ IV mais infusão a $10 \%$ de glicose $10 \mathrm{mg} / \mathrm{Kg}$ em cloreto de $\mathrm{Na}$ a $0,9 \%$, por uma hora. No outro grupo, preconizou-se o tratamento com frutose 1,6 difosfato, IV a $50 \mathrm{mg} / \mathrm{Kg}$ mais infusão a $10 \%$ da mesma solução $10 \mathrm{mg} / \mathrm{Kg}$ em cloreto de $\mathrm{Na}$ a $0,9 \%$, por uma hora.

O delineamento utilizado foi inteiramente casualizado com parcelas subdivididas, testando o fator grupo ou tratamento ( 2 níveis) nas parcelas e o fator tempo (4 níveis) nas subparcelas com cinco repetições dentro de cada tratamento (animais). Para os valores de teste de Fischer $(p<0,05)$, os efeitos de tempo e tratamento foram desdobrados, comparando as médias pelo teste de Tukey, cujo nível de significância foi 0,05. A analise estatística foi realizada no programa Statical Analysis System - SAS (1996).

\section{Resultados}

Nos resultados obtidos com esse trabalho relataram-se os sinais clínicos apresentados durante a intoxicação com N.oleander. Além disso, houve algumas diferenças em parâmetros laboratoriais e nos traçados eletrocardiográficos, nos diferentes períodos.

Os dois grupos $\mathrm{G} 1$ e $\mathrm{G} 2$ consumiram a totalidade da mistura de ração com folhas verdes de $\mathrm{N}$. oleander e os dois grupos apresentaram os mesmos sinais clínicos pós-intoxicação. Eles foram observados entre 30 a 90 minutos após o consumo, apresentaram sinais como: vômito, sialorréia, náuseas, apatia, conjuntiva ocular congesta, desidratação, dor abdominal, tremores, diarreia e tenesmo. Notaram-se em alguns animais inapetência, após o tratamento três animais do G2 diminuíram o consumo de ração no dia 
BELLODI, C., SOCHA, J.J.M. e HATAYDE, M.R. Intoxicação experimental de cães com folhas verdes de Nerium oleander e uso da frutose 1,6 difosfato e da glicose como tratamentos. PUBVET, Londrina, V. 8, N. 5, Ed. 254, Art. 1679, Março, 2014.

posterior. O aparecimento dos sinais clínicos se deu logo nas 4 primeiras horas do tratamento, sendo o primeiro sinal clínico apresentado foi o vômito.

Outras avaliações clínicas tais, como peso, temperatura, tempo de preenchimento capilar, cor das mucosas e frequência respiratória, não tiveram alterações importantes e mantiveram-se dentro dos parâmetros de normalidade para a espécie canina. A frequência cardíaca, dada em batimentos por minuto (bpm), não apresentou diferença significativa $(p \square 0,05)$ entre tratamentos, mas mostrou uma importante diminuição nos dois grupos em T4h.

$\mathrm{Na}$ interpretação e análise eletrocardiográfica foram encontrados diversos tipos de arritmias a partir do T4h até T24h, as quais se resolveram sem tratamento antiarrítmico nos dois grupos. As arritmias observadas foram bradicardia sinusal, bloqueios de segundo grau, complexos ventriculares prematuros, taquicardia ventricular e taquicardia sinusal.Nos exames laboratoriais não foi encontrado diferença significativa entre os parâmetros hematológicos e bioquímicos analisados.

\section{Discussão}

Neste trabalho foram usadas dosagens de folhas $0,25 \mathrm{~g} / \mathrm{Kg}$ do peso do animal, sem levar a óbito. Alguns estudos realizados em cães utilizando principalmente as folhas secas ou extratos isolou-se um dos princípios ativos (oleandrina), foi administrando em diferentes concentrações causando sintomas de intoxicação (TRAUTVETTER et al., 1969; SZABUNIEWICZ et al., 1971; LEHMANN, 1984; CLARK et al., 1991). Pesquisadores (SZABUNIEWICZ et al., 1971; GALEY, 1996), consideram o $N$. oleander como uma planta extremamente tóxica e dosagens de folhas $0,05 \mathrm{~g} / \mathrm{Kg}$ do peso do animal podem causar a morte. Citações na literatura descrevem doses letais com folhas secas ou fazendo referência a número de folhas utilizadas para intoxicar, sem ter considerado as concentrações dos princípios ativos (HENNING, 1932; REZAKHANI; MAHAN, 1994), entretanto estas informações suscitam muitas controvérsias e são consideradas inapropriadas devidas a que variam 
BELLODI, C., SOCHA, J.J.M. e HATAYDE, M.R. Intoxicação experimental de cães com folhas verdes de Nerium oleander e uso da frutose 1,6 difosfato e da glicose como tratamentos. PUBVET, Londrina, V. 8, N. 5, Ed. 254, Art. 1679, Março, 2014.

dependendo do tamanho das folhas e de outras variáveis como: estação do ano, estágio do desenvolvimento da planta e condições do meio ambiente (JOUBERT, 1989).Neste estudo buscamos mimetizar a intoxicação natural, nós nos propusemos reproduzir a intoxicação de uma forma mais natural possível, com a justificativa de que a planta tem o principio ativo a oleandrina e que na natureza ela esta composta por mais de 30 sustâncias potencialmente toxicas e esta disponível em jardines já que trata-se de uma planta ornamental.

Os sinais clínicos reportados neste trabalho são inespecíficos e podem ser confundidos com outros tipos de intoxicações, embora estes sinais ocorreram a pouco tempo após a ingestão das folhas. LANGFORD; BOOR (1996), mencionam em seu trabalho de revisão de literatura, sinais como: vômito diarréia, sialorréia, apatia, depressão do sistema nervoso central, hipotensão, membranas mucosas pálidas, tremores musculares, pulso irregular, diversas arritmias e coma, os quais foram observados também em nosso trabalho, mas sem ter consequências graves como: o coma ou óbito, provavelmente pela quantidade de toxina absorvida, como já foi descrito. Neste estudo clinicamente os animais que receberam a frutose 1,6 difosfato apresentaram uma melhora clinica na comparação com o grupo da glicose, fato evidenciado pelo retorno ao consumo de alimento novamente, observado em um numero maior de animais no Gll.

Uma variedade de alterações eletrocardiográficas são mostradas na intoxicação com $N$. oleander, incluindo mudanças na duração dos complexos e arritmias como: bradicardia, bloqueios de primeiro e segundo grau, complexos ventriculares prematuros, taquicardia ventricular, taquicardia sinusal e fibrilação entre outros, os quais foram evidenciados neste trabalho. Estas arritmias são originadas pelo alto grau de inibição da bomba $\mathrm{Na}+/ \mathrm{K}+$ ATPase nas fibras musculares cardíacas, condição que causa redução na atividade elétrica do miocárdio. A inibição da bomba $\mathrm{Na}+/ \mathrm{K}+$ ATPase pelos glicosídeos resulta em acúmulo intracelular de íons de sódio. Por sua vez permite o fluxo de íons de cálcio intracelular e a liberação de íons de cálcio ligados ao retículo sarcoplasmático. O incremento das concentrações livres de cálcio no 
BELLODI, C., SOCHA, J.J.M. e HATAYDE, M.R. Intoxicação experimental de cães com folhas verdes de Nerium oleander e uso da frutose 1,6 difosfato e da glicose como tratamentos. PUBVET, Londrina, V. 8, N. 5, Ed. 254, Art. 1679, Março, 2014.

sarcoplasma causa maior força de contração do miocárdio, o efeito inotrópico positivo (JOUBERT, 1989). Além disso, induz de forma indireta a diminuição da condução elétrica no coração, pelo incremento da tonicidade no nervo vago (KATZUNG; PARMLEY, 1998), que pode explicar a diminuição da frequência cardíaca no Gl em T4h e no Gll em T24h, e do aumento do intervalo Q-T evidenciados neste trabalho.Os compostos tóxicos da planta, por serem similares quimicamente com a digoxina e na farmacocinética, razão pela qual existem alguns fatores que alteram sua disponibilidade e que poderiam influenciar no grau de intoxicação do animal como: ser uma sustância pouco lipossolúvel que causaria deposição na gordura e maior tempo para sua eliminação, ela pode ter menor absorção ao ser administrada com o alimento, animais com hipoalbuminemia pode diminuir a união e ter maiores concentrações em sangue e a hipocalemia que diminuem a competição nos lugares de união e permitiria maiores quantidades séricas (MERRETT, 2000). É necessária a realização de outros estudos para conhecer as concentrações dos glicosídeos cardíacos na planta e suas doses letais em cães.

\section{Conclusão}

Com o presente estudo pode-se concluir que animais intoxicados com $0,25 \mathrm{~g} / \mathrm{Kg}$ de folhas verdes de Nerium oleander em única dose, causa sinais clínicos, alterações laboratoriais e mudanças no traçado do eletrocardiograma. O uso da frutose 1,6 difosfato e da glicose tiveram um comportamento similar para as variáveis estudadas durante o tempo experimental, excetuando uma melhora clinica observada no consumo do alimento, a qual foi melhor para a frutose 1,6 difosfato. O eletrocardiograma e mensuração da pressão fornecem uma avaliação do sistema cardiovascular, que mostra que as primeiras 24 horas nos animais intoxicados sofrem as alterações mais graves como hipotensão e arritmias. A intoxicação com $N$. oleander provocam vômito ao pouco tempo do consumo da planta o que tem como consequências diferentes graus de intoxicação. 


\section{Referências bibliográficas}

ASLANI, M.R.; MOVASAGHI, A.R.; JANATI-PIROUZ, H.; KARAZMA, M. Experimental oleander (Nerium oleander) poisoning in goats: a clinical and pathological study. Iranian Journal of Veterinary Research, Islamic republic of Iran, v. 8, p. 58-63, 2007.

ASLANI, M.R.; MOVASAGHI, A.R.; MOHRI, M.; ABBASIAN, A.; ZAREHPOUR M. Clinical and pathological aspects of experimental oleander (Nerium oleander) toxicosis in sheep. Veterinary Research Communications. Amsterdam, v. 28, p. 609-616. 2004.

BREWSTER, D. Herbal poisoning: a case report of a fatal yellow oleander poisoning in Solomon Islands. Annals of Tropical Paediatrics, London, v. 6, p. 289-291, 1986.

CAMPHAUSEN, C.; HAAS, N.A.; MATTKE, A.C. Successful treatment of oleander intoxication (cardiac glycosides) with digoxin-specific Fab antibody fragments in a 7-year-old child. Zeitschrift für Kardiologie, Darmstadt, v. 94, p. 817-823, 2005.

CHEUNG, K.; HINDS, J A.; DUFFY, P. Detection of poisoning by plant origin cardiac glycosides with the Abbott TDx analyzer. Clinical Chemistry, Baltimore, v. 35 p. 295-297, 1989.

CLARCK, R.; SELDEN, B.S.; CURRY, S.C. Digoxin - specific Fab fragments in the treatment of oleander toxicity in a canine model. Annals of Emergency Medicine,Lansing, v. 20, p. 1073 - 1077, 1991.

EVERIST,S.L. Apocynaceae In: Poisonous plants of Australia. Ed. Angus and Robertson. London, p. 77-89, 1981.

GALEY, F.D.; HOLSTEGE, D.M.; PLUMLEE, K.H. Diagnosis of oleander poisoning in livestock. Journal Veterinary Diagnostic Investigation, Columbia, v. 8, p. 358-364, 1996.

HUGHES, K.J. DART, A.J.; HOGSON D.R. Suspected Nerium oleander poisoning in a horse, Australian Veterinary Journal, Sidney, v. 80, p.412-415, 2002.

IBRAHIM, A.; OMAR, Z.; NAWAL, A.; ANWAR, M. A fatal case of oleandrin poisoning. Forensic Science International, Lausanne, v. 179, p.31-36, 2008.

JORTANI, S.A.; HELM, R.A.; VALDES, R. Inhibition of $\mathrm{Na}, \mathrm{K}-\mathrm{ATP}$ ase by oleandrin and oleandrigenin, and their detection by digoxin immunoassays. Clinical Chemistry,Baltimore, $v$. 42, p. 1654-1658, 1996.

JOUBERT,J.P.J. Cardiac glicosides. In: CHEEKE, P.R. (Ed.), In: Toxicants of Plant Origin, $v$ II.CRC Press, Boca Raton, p.61-96, 1989.

KATZUNG, B.G.: PARMLEY, W.W. Cardiac glycosides and other drugs used in

congestive heart failure. Ed, Basic and clinical pharmacology. Beirut, p. 197-216,1998.

KNIGHT, AP. Oleander poisoning. Compendium on Continuing Education for The Practicing Veterinarian, Princeton, v. 10, p. 262-263,1988.

LANGFORD, S.D.; BOOR, P.J. Oleander toxicity: an examination of human and animal exposures. Toxicology, Amsterdam, v. 109, p. 1-13, 1996.

LYNN, M., AND SAFDAR, A. An overview of potentially life-threatening poisonous plants in dogs and cats. Journal of Veterinary Emergency and Critical Care, San Antonio, v. 16, n.1, p. 25-33, 2006.

LEHMANN, VON H.D. Zur wirkung pflanzlicher glycoside auf widerstandsgefabe und kapazzitatsgefabe. Arzneim Forsch. Berlin, v.34, p.423-429, 1984.

MARIN, M.; ARCE, A.; MARTINEZ, A.; MATEU, J. Diagnóstico de intoxicación por Nerium oleander: un único método analítico es suficiente. Farmacia Hospitalaria, Madrid, $\vee 31$, 2007. 
MARKOV, A.K.; PAYMENT, M.F.; HUME, A.S.; RAO, M.R.; MARKOV, M.A.; SKELTO, T.N.; LEHAN, P.H. Fructose-1,6-diphosphate in the treatment of oleander toxicity in dogs. Veterinary and Human Toxicology, Manhathan, v. 41, p. 9-15. 1999.

MARTINEZ, B.R.; FERRANDO, R.; MILARA, J.; SOLER. E. Intoxicación por Nerium oleander (baladre) de los casos clínicos. Farmacia Hospitalaria, Madrid, v. 31, 2007.

MERRETT, D. Digoxin therapy. Australian Veterinary Journal. Victoria, v.78, p. 612-615, 2000.

PLUMB, D.C. Veterinary drug handbook. 3nd ed., Iowa: Iowa State University Press,p. 228233, 1999.

REZAKHANI, A.; MAHAM, M. Oleander poisoning in cattle of the fars province, Iran.Veterinary Human Toxicol., v. 34, p, 549, 1992.

SZABUNIEWICZ, M.; MCCARDY, W.L.; CAMP, B.J. Treatment of experimentally

induced oleander poisoning. Archives Internationales de Pharmacodynamie et de Thérapie, Gent, v. 189, p. 12-21, 1971.

SOTO-BLANCO, B.; FONTENELE-NETO, N.D.; SILVA D.M.; REIS P.F.C.C.;

NOBREGA, J.E. Acute cattle intoxication from Nerium oleander pods. Tropical Animal Health and Production, Edinburgh, v. 38, p. 451-454, 2006.

TRAUTVETTER, E.; KASBOHM, C.; WERNER, J. Oleander poisoning with respiratory AV block in a dog. Berlin Munich Tierarztl Wochenschr. Berlin, v. 15, p. 306-308, 1969. 